\title{
Similarity Word-Sequence Kernels for Sentence Clustering
}

Jesús Andrés-Ferrer, Germán Sanchis-Trilles, and Francisco Casacuberta

\author{
Instituto Tecnológico de Informática \\ Departamento de Sistemas Informáticos y Computación \\ Universidad Politécnica de Valencia \\ \{jandres,gsanchis,fcn\}@dsic.upv.es
}

\begin{abstract}
In this paper, we present a novel clustering approach based on the use of kernels as similarity functions and the $C$-means algorithm. Several word-sequence kernels are defined and extended to verify the properties of similarity functions. Afterwards, these monolingual wordsequence kernels are extended to bilingual word-sequence kernels, and applied to the task of monolingual and bilingual sentence clustering. The motivation of this proposal is to group similar sentences into clusters so that specialised models can be trained for each cluster, with the purpose of reducing in this way both the size and complexity of the initial task. We provide empirical evidence for proving that the use of bilingual kernels can lead to better clusters, in terms of intra-cluster perplexities.
\end{abstract}

\section{Introduction}

Text categorisation [1] is the task of finding the class to which a given document belongs to. The categories or classes in which a document can be classified are known beforehand, and, usually, a database of documents with their corresponding category is enough for training an automatic categorisation system. Several approaches have been applied to text categorisation, ranging from naive Bayes classifiers [1] to support vector machines (SVM) 23].

A variant of text categorisation is the text clustering task. Unlike text categorisation, in text clustering we do not know the classes into which the documents should be classified, which means that the only data available is a database of documents without class information. Therefore, text clustering is entailed as a more difficult task than text categorisation. Several attempts have been made in text clustering. For instance, in [4] several kernel-based text categorisation techniques are adapted to text clustering by using the $C$-means algorithm.

An especially appealing problem in document clustering is sentence clustering, in which each document is made up of only one single sentence. This problem has been receiving special attention in the natural language processing (NLP) community since it allows for training specific models for each of the obtained clusters, leading to more task-focused models [56]. Moreover, sentence clustering can also be of interest for kernel-based methods when applied to NLP tasks, such as done for text recognition [7] or statistical machine translation [89. In

E.R. Hancock et al. (Eds.): SSPR \& SPR 2010, LNCS 6218, pp. 610-619. 2010.
(c) Springer-Verlag Berlin Heidelberg 2010 
these scenarios, kernel methods often suffer scalability problems, and sentence clustering is a natural way in which the training data can be divided so as to obtain smaller (but more specific) models.

Throughout literature, the "sum-of-squares" cost for a given data set, forms the basis for a number of clustering methods 10. The aim of such clustering algorithms is to partition a data set of $N$ samples, $\boldsymbol{x}_{1}, \ldots, \boldsymbol{x}_{N}$, into $C$ clusters, so as to minimise the intra-cluster mean squared error. The standard algorithm, also known as Lloyd's algorithm or $C$-means [11, relies on assigning each data point $\boldsymbol{x}_{n}$ to the cluster with the closest mean. Once all the data points have been assigned, the means of each cluster are updated according to the samples contained within it. Then, the data points are reassigned to the cluster with the closest mean, and this procedure is repeated iteratively until no data points are changed. The $C$-means algorithm is considered a fast clustering method because it is a sub-optimal algorithm that does not require the computation of the distance matrix between all samples. One of the disadvantages of $C$-means is that it is unable to find suitable clusters whenever the given data are not linearly separable, leading to degenerated solutions in which the number of clusters computed exceeds the desirable amount. In order to circumvent this problem, M. Girolami proposed in 12 an extension of $C$-means that relies on a transformation of the original sample $\boldsymbol{x}$ into a higher-dimensionality feature space $\phi(\boldsymbol{x})$. Although such proposal is based on the computation of Mercer kernels [13], it still relies on the distance metric of the original $C$-means algorithm.

Kernel methods have attracted much interest since they were introduced by V. Vapnik [14. Traditionally being applied to classification problems in the form of Support Vector Machines (SVM) 1516], kernel methods rely on the idea of establishing a mapping from the current feature space to a higher-dimensionality feature space, with the purpose of achieving linear separability among classes which are non-separable in the current feature space. Under this perspective, a kernel function between two data points is defined as

$$
k\left(\boldsymbol{x}, \boldsymbol{x}^{\prime}\right)=\phi(\boldsymbol{x})^{T} \phi\left(\boldsymbol{x}^{\prime}\right),
$$

where $\boldsymbol{x}$ and $\boldsymbol{x}^{\prime}$ are the data points considered and $\phi(\boldsymbol{x})$ is the mapping function to a higher-dimensionality feature space. From the definition above, it is clear that a kernel is a symmetric function, i.e., $k\left(\boldsymbol{x}, \boldsymbol{x}^{\prime}\right)=k\left(\boldsymbol{x}^{\prime}, \boldsymbol{x}\right)$.

Given that kernels are implemented as a dot product in a metric space, some kernels can also be used to measure the similarity (or distance) between the data points considered and are appropriate for direct application within distancebased clustering algorithms.

In this paper, we present a new approach for using similarity Mercer kernels for clustering based on the $C$-means algorithm. This new approach is evaluated in practise under the scope of sentence clustering and bilingual sentence clustering.

This paper is structured as follows: in the next Section, we extend the $C$ means algorithm for using kernel methods. In Section 3 word-sequence kernels are introduced, and they are extended to bilingual word-sequence kernels in the following Section. The empirical results are gathered in Section 5, and concluding remarks are discussed in Section 6 . 


\section{Kernel-Based $C$-Means Clustering}

The $C$-means algorithm [11] seeks to minimise the sum-of-squares distance from each sample to the centre of the cluster it belongs to. Given a number of categories $C$, the algorithm finds a local optimum for for the following minimisation

$$
\hat{\boldsymbol{z}}=\underset{\boldsymbol{z}}{\arg \min }\left\{\frac{1}{N} \sum_{c=1}^{C} \sum_{n=1}^{N} z_{n c} \mathrm{~d}\left(\boldsymbol{x}_{n}, \boldsymbol{m}_{c}\right)\right\},
$$

where $z_{n c}=1$ if $\boldsymbol{x}_{n}$ belongs to the $c$-th cluster and 0 otherwise, and with $\boldsymbol{m}_{c}$ being the centre of the $c$-th cluster, $\boldsymbol{m}_{c}=N_{c}^{-1} \sum_{n=1}^{N} z_{n c} \boldsymbol{x}_{n c}$, where $N_{c}$ stands for the number of samples in the $c$-th cluster, i.e., $N_{c}=\sum_{n=1}^{N} z_{n c}$. The function $\mathrm{d}\left(\boldsymbol{x}_{n}, \boldsymbol{m}_{c}\right)$ is a distance function between the sample $\boldsymbol{x}_{n}$ and the centre $\boldsymbol{m}_{c}$, usually the euclidean distance

$$
\mathrm{d}\left(\boldsymbol{x}_{n}, \boldsymbol{m}_{c}\right)=\left(\boldsymbol{x}_{n}-\boldsymbol{m}_{c}\right)^{T}\left(\boldsymbol{x}_{n}-\boldsymbol{m}_{c}\right) .
$$

The distance used by the $C$-means algorithm can either be a semi-metric or a metric, depending on whether the triangle inequality is verified or not.

In [12] $C$-means was extended with the help of Mercer kernels by changing the distance and the centres of the standard algorithm, so that it can better handle non linearly-separable data. The distance proposed in 12 is given by

$$
\mathrm{d}\left(\boldsymbol{x}_{n}, \boldsymbol{m}_{c}\right)=\left(\phi\left(\boldsymbol{x}_{n}\right)-\boldsymbol{m}_{c}\right)^{T}\left(\phi\left(\boldsymbol{x}_{n}\right)-\boldsymbol{m}_{c}\right),
$$

with $\boldsymbol{m}_{c}=N_{c}^{-1} \sum_{n=1}^{N} \boldsymbol{z}_{n c} \phi\left(\boldsymbol{x}_{n c}\right)$.

Since kernel functions are symmetric, they only need to verify two more conditions to be a semi-metric distance. Therefore, the kernel itself can be used as the distance inside the $C$-means algorithm. Moreover, if a given kernel also verifies the triangle inequality, then the kernel itself can be used as a metric distance. However, many kernels are more naturally redefined as similarity functions instead of distances. Given a distance, a similarity can be defined and vice-versa. In such case, $C$-means can be re-defined in terms of similarities as follows

$$
\hat{\boldsymbol{z}}=\underset{\boldsymbol{z}}{\arg \max }\left\{\frac{1}{N} \sum_{c=1}^{C} \sum_{n=1}^{N} z_{n c} \mathrm{~s}\left(\boldsymbol{x}_{n}, \boldsymbol{m}_{c}\right)\right\},
$$

with $\boldsymbol{m}_{c}=N_{c}^{-1} \sum_{n=1}^{N} z_{n c} \phi\left(\boldsymbol{x}_{n c}\right)$, and where $\mathrm{s}\left(\boldsymbol{x}_{n}, \boldsymbol{m}_{c}\right)=\phi\left(\boldsymbol{x}_{n c}\right)^{T} \boldsymbol{m}_{c}$ is assumed to be a (semi-)metric kernel, i.e., a kernel that measures the similarity generated by a (semi-)metric distance. In this work, several (semi-)metric kernels are proposed so that they can be used within the similarity version of $C$-means. However, the approaches in Eqs. (2) and (5) could be related in some way.

\section{Word-Sequence Kernels}

Recently, Word-sequence Kernels (WSK) were introduced in [17]. The main purpose of WSK is to compute document similarity based on matching nonconsecutive sequences of words. WSK are defined as a mapping $\Sigma^{n} \rightarrow \mathbb{R}^{|\Sigma|^{n}}$, 
where $n$ stands for the maximum length of the segment to be considered. For a given order $n$ and document pair $\left(\boldsymbol{x}, \boldsymbol{x}^{\prime}\right)$, we define the following kernel

$$
K_{n}\left(\boldsymbol{x}, \boldsymbol{x}^{\prime}\right)=\sum_{u \in \Sigma^{n}}|\boldsymbol{x}|_{u}\left|\boldsymbol{x}^{\prime}\right|_{u},
$$

where $|\boldsymbol{x}|_{u}$ stands for the number of occurrences of $u$ in document $\boldsymbol{x}$. In their work, [17] reported interesting improvements by using WSK when applied to text categorisation tasks. However, the best results were achieved using a small order, $n=2$.

Although the kernel defined in Eq. (6), is intuitively correct, it does not verify some of the requirements to be a semi-metric similarity. Hence, we also define the kernel $K_{n}^{1}$ as follows:

$$
K_{n}^{1}\left(\boldsymbol{x}, \boldsymbol{x}^{\prime}\right)=\sum_{u \in \Sigma^{n}} 1_{u}(\boldsymbol{x}) 1_{u}\left(\boldsymbol{x}^{\prime}\right)
$$

where $1_{u}(\boldsymbol{x})=1$ if $u$ appears in $\boldsymbol{x}$, and 0 otherwise.

The intuitive justification for defining this last kernel can be explained with a small example. We start by defining the following strings:

$$
\begin{array}{ll}
s_{1}=\{a b c b\} & s_{2}=\{a b a b\} \\
s_{3}=\{a b e b\} & s_{4}=\{a b c b a b\}
\end{array}
$$

One would state that $\boldsymbol{s}_{1}$ is as similar to $\boldsymbol{s}_{2}$ as to $\boldsymbol{s}_{3}$, under the prior assumption of a Levenshtein distance. However, $K_{2}\left(\boldsymbol{s}_{1}, \boldsymbol{s}_{2}\right)=2$ and $K_{2}\left(\boldsymbol{s}_{1}, \boldsymbol{s}_{3}\right)=1$. This is exactly the reason why we introduce kernel $K_{2}^{1}$, since $K_{2}^{1}\left(s_{1}, s_{2}\right)=K_{2}^{1}\left(s_{1}, s_{3}\right)=$ 1. On the other hand, the similarity of $s_{1}$ with itself is $K_{2}^{1}\left(s_{1}, s_{1}\right)=3$, which is the same than that of $\boldsymbol{s}_{1}$ with $\boldsymbol{s}_{4}, K_{2}^{1}\left(\boldsymbol{s}_{1}, \boldsymbol{s}_{4}\right)=3$. This is because the kernel $K_{2}^{1}$ is a pseudo-metric similarity. It is worth noting that $K_{n}$ is not a pseudo-metric, which implies that a given element, as in the example $\boldsymbol{s}_{4}$, may be more similar to a given element, such as $\boldsymbol{s}_{1}$, than the element itself, i.e. $K_{2}\left(\boldsymbol{s}_{1}, \boldsymbol{s}_{4}\right)=4>$ $K_{2}\left(s_{1}, s_{1}\right)=3$. Such problem cannot be underestimated, since it can imply that $C$-means will fail to converge.

To solve this undesirable property, $K_{n}^{1}$ is redefined using a normalisation score depending on the different number of $n$-grams of the sample, i.e.,

$$
\hat{K}_{n}^{1}=\sum_{u \in \Sigma^{n}} \frac{1_{u}(\boldsymbol{x})}{\sqrt{\sum_{v \in \Sigma^{n}} 1_{v}(\boldsymbol{x})}} \frac{1_{u}\left(\boldsymbol{x}^{\prime}\right)}{\sqrt{\sum_{v \in \Sigma^{n}} 1_{v}\left(\boldsymbol{x}^{\prime}\right)}}
$$

The kernel defined in Eq. (8) solves the previously outlined problem, i.e. the similarity of $s_{1}$ with itself is $\hat{K}_{2}^{1}\left(s_{1}, s_{1}\right)=1$, which is larger than the similarity of $s_{1}$ with $s_{4}, \hat{K}_{2}^{1}\left(s_{1}, s_{4}\right)=0.866$. With this last kernel, we achieve a very desirable property for its use within $C$-means, i.e., a given element achieves maximum similarity only when it is compared with itself.

Similarly, we also redefine the kernel $K_{n}$,

$$
\hat{K}_{n}\left(\boldsymbol{x}, \boldsymbol{x}^{\prime}\right)=\sum_{u \in \Sigma^{n}} \frac{|\boldsymbol{x}|_{u}}{\sqrt{\sum_{v \in \Sigma^{n}}|\boldsymbol{x}|_{v}}} \frac{\left|\boldsymbol{x}^{\prime}\right|_{u}}{\sqrt{\sum_{v \in \Sigma^{n}}\left|\boldsymbol{x}^{\prime}\right|_{v}}}
$$


However, the re-normalised version of $K_{n}, \hat{K}_{n}$, only reduces the cases in which the problem of not being a semi-metric can appear, but it does not solve it.

Given the definition in Eq. (9), a WSK $\bar{K}_{n}$ is defined as

$$
\bar{K}_{n}\left(\boldsymbol{x}, \boldsymbol{x}^{\prime}\right)=\sum_{i=1}^{n} \hat{K}_{i}\left(\boldsymbol{x}, \boldsymbol{x}^{\prime}\right)
$$

Analogously as done above, the kernel defined in Eq. (8) is extended to $\bar{K}_{n}^{1}$.

\section{Bilingual Word-Sequence Kernels}

In [17], cross-lingual WSK were also defined, by first defining a soft matching WSK and assuming that the samples being considered (i.e. $\boldsymbol{x}$ and $\boldsymbol{x}^{\prime}$ ) belonged to different languages. In this context, soft matching refers to a probabilistic matching, i.e. a matching that does not require both samples to have exactly identical parts. By doing so, they were able to find similarities between documents written in different languages.

Our purpose, however, is not to perform cross-lingual classification (or clustering). Our case is different, since we assume that we have a sentence-aligned bilingual corpus and we intend to cluster the data by taking into account such bilingual information. Hence, we need to define a bilingual WSK (BWSK), which can be easily extended from the one defined in Eq. (10) by taking into account two different vocabularies, namely $\Sigma$ for the source language and $\Delta$ for the target language. Let be $\boldsymbol{w}=\{\boldsymbol{x}, \boldsymbol{y}\}$ a bilingual sentence pair, where $\boldsymbol{x}$ is the sentence belonging to the source language and $\boldsymbol{y}$ is the sentence belonging to the target language. Then, a BWSK can be defined as

$$
B_{n}\left(\boldsymbol{w}, \boldsymbol{w}^{\prime}\right)=K_{n}\left(\boldsymbol{x}, \boldsymbol{x}^{\prime}\right)+K_{n}\left(\boldsymbol{y}, \boldsymbol{y}^{\prime}\right)=\sum_{u \in \Sigma^{n}}|\boldsymbol{x}|_{u}\left|\boldsymbol{x}^{\prime}\right|_{u}+\sum_{v \in \Delta^{n}}|\boldsymbol{y}|_{v}\left|\boldsymbol{y}^{\prime}\right|_{v}
$$

Note that $B_{n}\left(\boldsymbol{w}, \boldsymbol{w}^{\prime}\right)$ is a kernel because it can be expressed as the sum of two kernels, which is a valid kernel composition rule.

As done for the monolingual case, we can also define $B_{n}^{1}\left(\boldsymbol{w}, \boldsymbol{w}^{\prime}\right)$; and all its extensions: $\hat{B}_{n}^{1}\left(\boldsymbol{w}, \boldsymbol{w}^{\prime}\right), \bar{B}_{n}^{1}\left(\boldsymbol{w}, \boldsymbol{w}^{\prime}\right) ;$ and $\hat{B}_{n}\left(\boldsymbol{w}, \boldsymbol{w}^{\prime}\right), \bar{B}_{n}\left(\boldsymbol{w}, \boldsymbol{w}^{\prime}\right)$.

\section{Experiments}

We ran most of our clustering experiments on the BTEC (Basic Travel Expression Corpus), which is the corpus provided for the IWSLT 1 statistical machine translation campaign. The BTEC corpus includes several bilingual, sentencealigned sub-corpora, among which we selected the Chinese-English one. The figures of this corpus are summarised in Table 1. Prior to performing clustering on the data, all English words were lowercased. This was not necessary on the Chinese side since Chinese has no case information.

${ }^{1}$ http://mastarpj.nict.go.jp/IWSLT2009/ 
The problem of automatically measuring the quality of the produced clusters was addressed by means of the perplexity concept. In natural language procesing (NLP) the perplexity of a test set $\left(\boldsymbol{w}=w_{1}, \ldots, w_{L}\right)$ is defined as follows:

$$
\operatorname{ppl}(\boldsymbol{w})=2^{\frac{1}{L} \log _{2} p(\boldsymbol{w})},
$$

where $p(\boldsymbol{w})$ is the probability of the test set accordingly to a language model. The intuitive meaning of perplexity is the average number of words that can follow a given word, according to a given language model. For instance, if the perplexity for a given data set is 28 , it means that in order to predict the word which follows a given prefix, a total average of 28 different words should be taken into account. Hence, the perplexity for a given data set according to the language model trained on that same data is a measure of how compact (i.e. not sparse) the data is. We will be assessing the quality of the clusters using the intra-cluster perplexity (IC-PPL) average, measured on the English data, given by

$$
p p l_{\text {avg }}=2^{\sum_{c=1}^{C} \frac{1}{C} \frac{1}{W_{c}} \log _{2} p(c)},
$$

where $p(c)$ is the probability of the samples of cluster $c$ according to the language model estimated on that same cluster; $W_{c}$ is the total number of words in the sentences belonging to the cluster $c$; and $C$ is the total number of clusters. Since we will be computing clusters using the kernels proposed in Secs. 3and 4 with the order $n$ ranging from 1 up to 4; we decided to compute IC-PPL based on a 5-gram language model computed using SRILM toolkit [18]. Furthermore, since cluster sizes dropped to less than 1000 sentences in some cases, we decided to smooth these models with the interpolated version of Knesser-Ney smoothing [19. For consistency purposes, the perplexities listed in Table 1 are also smoothed with the same smoothing.

Table 1. Statistics of the BTEC corpus. $K$ stands for thousands of elements

\begin{tabular}{l|cccc|} 
Language & N. Sentences Running words & Vocabulary & Perplexity \\
\hline Chinese & $20 \mathrm{~K}$ & $172 \mathrm{~K}$ & 8428 & 24.3 \\
English & $20 \mathrm{~K}$ & $183 \mathrm{~K}$ & 7298 & 20.8 \\
\hline
\end{tabular}

In preliminary investigation, we also researched the use of average edit distance from each sentence of a cluster to all other sentences in the same cluster as quality metric, but the differences reported were similar to those reported by IC-PPL, which is much faster to compute.

One way to reduce the computational requirements of our clustering algorithm without any loss of information was to remove all singletons, since their effect on the calculation of the kernels is minimum, if any. For a similar reasons, we also decided to remove stop words, since if a word appears in almost every sentence, then its discriminative capacity should not be very high either.

We computed 2 to 20 clusters of the training data, with steps of two, for all kernels described in Sections 3 and 4 . Since the $C$-means algorithm needs a 

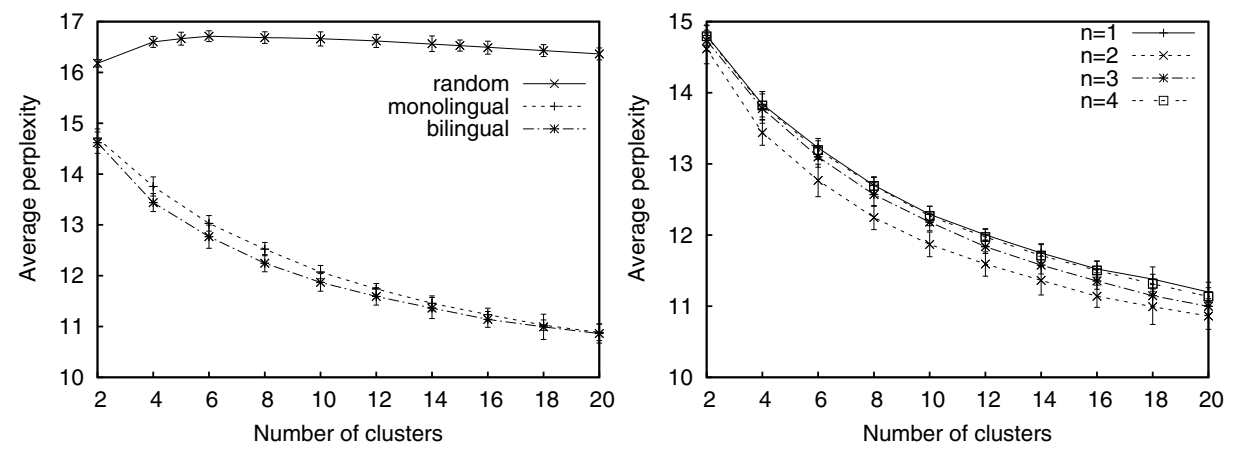

Fig. 1. Clusterings for the BTEC corpus. Left: IC-PPL for random, $\hat{K}_{2}^{1}$ (monolingual) and $\hat{B}_{2}^{1}$ (bilingual). Right: IC-PPL for $\bar{B}_{1}^{1}, \bar{B}_{2}^{1}, \bar{B}_{3}^{1}$ and $\bar{B}_{4}^{1}$ (right).

random initialisation, we performed 20 repetitions of each experiment and report the average and the confidence interval at $95 \%$.

Let us now analyse the results in detail. First, we show in Figure 1 the performance of the $\bar{K}_{2}^{1}$ and $\bar{B}_{2}^{1}$ kernels when used in $C$-means, as compared to a random clustering. Other proposed kernels, such as e.g. $\bar{K}_{1}^{1}$ or $\bar{B}_{3}$, present a similar behaviour, and are not shown here for the sake of simplicity. The first thing to be noted is that IC-PPL stays almost unchanged for every number of clusters considered in the case of random clustering, whereas for the kernel-clusterings IC-PPL drops logarithmically when increasing the number of clusters. This fact was actually expected: if we consider $20 \mathrm{~K}$ clusters (as many as sentences in the corpus), IC-PPL will eventually drop to 1 . However, since we are only considering up to 20 clusters and an average of 1000 sentences are included into each cluster, perplexity will only drop when such grouping is done in an informed way. It seems that considering bilingual information has beneficial effects since BWSK lead to smaller IC-PPL than regular WSK.

As for the effect of considering different $n$ orders, in Figure 1 we show the result of comparing $\bar{B}_{1}^{1}, \bar{B}_{2}^{1}, \bar{B}_{3}^{1}$ and $\bar{B}_{4}^{1}$. Again, other kernels such as the monolingual ones, perform similarly and are omitted for clarity. We can see that the best performance is given by $\bar{B}_{2}^{1}$, and that increasing the order of $n$ above 2 does not provide further improvements, but rather has a degrading effect on IC-PPL. This fact is consistent with what [17] reported for document classification tasks.

In order to check the scalability of the results reported on the BTEC corpus to other larger corpora, we also performed some experiments on a reduced version of the Spanish-English Europarl corpus [20]. Such version was restricted to maximum sentence length of $20($ Euro $<20)$, for both English and Spanish. The statistics of this corpus are summarised in Table 2, As for the BTEC corpus, we will measure cluster quality with IC-PPL measured on the English side.

The first thing that we notice when observing Figure 2 is that the monolingual and the bilingual kernel clusterings behave similarly, as with the BTEC corpus. This is probably due to the fact that, once the monolingual information 

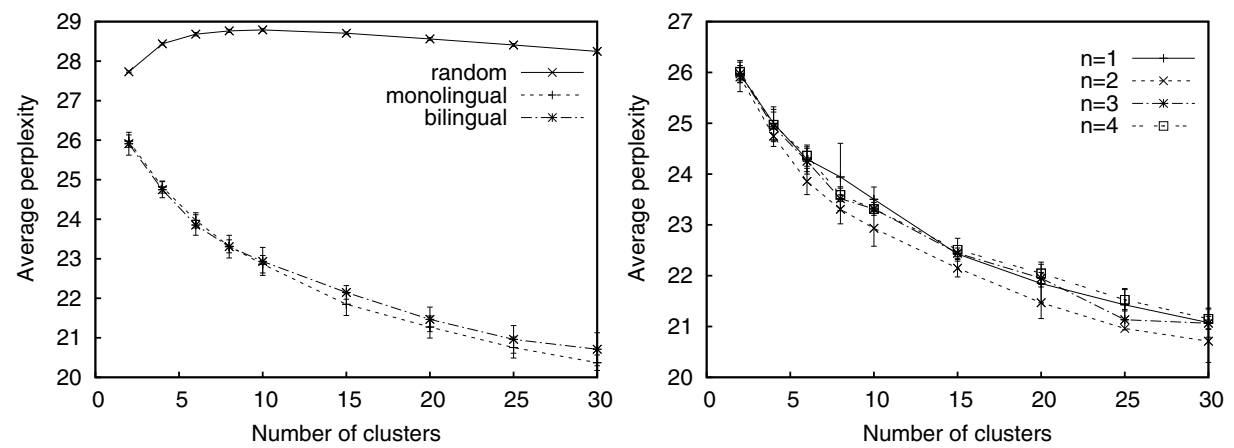

Fig. 2. Clusterings for Europarl with maximum sentence length of 20 (Euro $<20)$. Left: IC-PPL for random, $\bar{K}_{2}^{1}$ and $\bar{B}_{2}^{1}$. Right: IC-PPL for $\bar{B}_{1}^{1}, \bar{B}_{2}^{1}, \bar{B}_{3}^{1}$ and $\bar{B}_{4}^{1}$.

Table 2. Statistics of the Spanish-English Europarl corpus, when restricted to maximum sentence length of $20 . K$ stands for thousands of elements.

\begin{tabular}{l|cccc|} 
Language & N. Sentences Running words & Vocabulary perplexity \\
\hline Spanish & $312 \mathrm{~K}$ & $4.0 \mathrm{M}$ & $58 \mathrm{~K}$ & 28.2 \\
English & $312 \mathrm{~K}$ & $3.9 \mathrm{M}$ & $37 \mathrm{~K}$ & 26.7 \\
\hline
\end{tabular}

is added to the cluster, the bilingual information is not able to produce a further refinement over the initial clusters. Nevertheless, thought not statistically significant, it can be observed that for small number of clusters, the bilingual information does seem to help, but as the number of clusters increase the bilingual information tends to confuse the clustering algorithm. These results, which are similar to the results observed with BTEC, suggest that bilingual information only helps when there is a large amount of samples within a cluster. This can be due to the fact that the smaller the clusters, the more focused they become in specific word-sequences, and the more easily extra-cluster information can distort them. When dealing with large cluster sizes, however, introducing bilingual information may help to group word-sequences which are not so similar in the English side, but appear more similar in their bilingual counterpart.

As for increasing the order of $n$ in the Euro $<20$ corpus, a similar behaviour as for BTEC is be observed in Figure 2. Similarly to the results shown above, $\bar{B}_{2}^{1}$ seems to be the best performing kernel in terms of IC-PPL. In order to understand the reason why $n=2$ is the best performing kernel-family, let us have a closer look at some statistics of the corpora considered. Looking at Table 3 , it is quite obvious why increasing the order of $n$ above 2 does not provide any improvements: only $10 \%$ of the trigrams and $5 \%$ of the 4-grams appear more than twice. This means that such features, when introduced into the clustering algorithm via WSK or BWSK will most likely only introduce noise.

As for the difference between the families of kernels defined by $|\boldsymbol{x}|_{u}$ and $1_{u}(\boldsymbol{x})$, our experiments show that they are indistinguishable according to IC-PPL. This 
Table 3. Statistics of the IWSLT and Euro $<20$ in terms of singletons and doubletons. Single stands for singletons and double for doubletons. All data are in \%.

\begin{tabular}{l|cc|cc|cc|cc|} 
& \multicolumn{2}{|c|}{ 1-grams } & \multicolumn{2}{|c|}{$\begin{array}{c}\text { 2-grams } \\
\text { Corpus }\end{array}$} & \multicolumn{2}{|c|}{$\begin{array}{c}\text { 3-grams } \\
\text { single double }\end{array}$} & \multicolumn{2}{c|}{$\begin{array}{c}\text { 4-grams } \\
\text { single }\end{array}$} \\
\hline BTEC & 43.8 & 14.0 & 65.3 & 13.6 & 79.0 & 10.5 & 87.5 & 7.5 \\
Euro $<20$ & 36.7 & 13.3 & 62.7 & 13.3 & 78.9 & 9.8 & 88.4 & 6.2 \\
\hline
\end{tabular}

is due to the fact that, although the theoretical motivation is clear as seen in Section 3 , in practise it is not very often the case that a given $n$-gram occurs more than once within a single sentence - not for unigrams and even less for bigrams. In fact, nearly no bigram happens twice in a single sentence once stopwords have been removed. This implies that $K_{n}$ is practically equivalent to $K_{n}^{1}$ (and all the variations thereof).

\section{Conclusions and Future Work}

In this work, we have proposed the direct use of kernels as similarity measure, and applied it to the specific case of sentence clustering via $C$-means. Specifically, we have described several families of kernels suitable for this task, and shown that the $\bar{B}_{2}$ and $\bar{B}_{2}^{1}$ kernels are the ones which perform the best. Although for other corpora it might be beneficial to increase the order of $n$, such corpora should be less sparse if improvements are to be expected. It is also observed that, in order to take full advantage of bilingual information, cluster sizes need to be larger.

As most of the cluster quality measures, such as cluster sparseness, IC-PPL does not provide any insight towards deciding the optimal number of clusters, C. For finding the optimal number of cluster, a possiblity is to use the bayesian scheme proposed in [12].

Given the generality provided by using kernels as similarity measure, the $C$ means algorithm used in this paper can be easily extended by just adding more components while sticking to the kernel composing rules. In this way, we plan to introduce other features inherent to NLP tasks, such as part-of-speech tags, automatic word classes, $n$-gram probability, or even bilingual lexicon probability for the case of bilingual kernels. We plan to address these issues in future works.

\section{Acknowledgements}

This paper is based upon work supported by the EC (FEDER, FSE), the Spanish Government (MICINN, MITyC, "Plan E" under grants MIPRCV "Consolider Ingenio 2010", iTrans2 TIN2009-14511 and erudito.com TSI-020110-2009-439) and the Generalitat Valenciana (grant Prometeo/2009/014).

The authors would also like to thank the anonimous reviewers for their constructive and detailed comments. 


\section{References}

1. McCallum, A., Nigam, K.: A comparison of event models for naive bayes text classification. In: Proc. of AAAI/ICML 1998 Workshop on Learning for Text Categorization, pp. 41-48. AAAI Press, Menlo Park (1998)

2. Joachims, T.: Text categorisation with support vector machines: learning with many relevant features. In: Proceedings of 10th ECML, pp. 137-142 (1998)

3. Lodhi, H., Saunders, C., Shawe-Taylor, J., Cristianini, N., Watkins, C.J.C.H.: Text classification using string kernels. JMLR 2, 419-444 (2002)

4. Karatzoglou, A., Feinerer, I.: Text clustering with string kernels in r. In: Proc. of the 30th Annual Conference of the Gesellschaft für Klassifikation e.V., Freie Universität Berlin (March 2006)

5. Sanchis-Trilles, G., Cettolo, M.: Online language model adaptation via n-gram mixtures in statistical machine translation. In: Proc. of 14th Annual Conference of the European Association for Machine Translation, Saint-Raphaël, France, (May $27-28,2010)$

6. Lagarda, A., Juan, A.: Topic detection and classification techniques. In: WP4 deliverable, TransType2 (2003)

7. Cortes, C., Mohri, M., Weston, J.: A general regression technique for learning transductions. In: Proc. of 22nd. ICML, pp. 153-160. ACM, NY, USA (2005)

8. Serrano, N., Andrés-Ferrer, J., Casacuberta, F.: On a kernel regression approach to machine translation. In: IbPRIA 2009. LNCS, vol. 5524, pp. 394-401. Springer, Heidelberg (2009)

9. Szedmak, Z.W.S.T.: Kernel regression based machine translation, pp. 185-188. Association for Computational Linguistics (2007)

10. Jain, A.K., Dubes, R.C.: Algorithms for clustering data. Prentice-Hall, Inc., Upper Saddle River (1988)

11. Lloyd, S.P.: Least squares quantization in pcm. IEEE Transactions on Information Theory 28(2), 129-137 (1982)

12. Girolami, M.: Mercer kernel based clustering in feature space. IEEE Transactions on Neural Networks (2001)

13. Mercer, J.: Functions of positive and negative type and their connection with the theory of integral equations. Philosophical Transactions of the Royal Society London (A) 209, 415-446 (1909)

14. Vapnik, V.: The Nature of Statistical Learning Theory. Springer, New York (1995)

15. Boser, B., Guyon, I., Vapnik, V.: A training algorithm for optimal margin classifiers. In: Annual Workshop on Computational Learning Theory, pp. 144-152 (1992)

16. Cortes, C., Vapnik, V.: Support-vector networks. Machine Learning 20(3), 273-297 (1995)

17. Cancedda, N., Gaussier, E., Goutteand, C., Renders, J.: Word-sequence kernels. Journal of Machine Learning Research 3, 1059-1082 (2003)

18. Stolcke, A.: SRILM - an extensible language modeling toolkit. In: Proc. of ICSLP 2002, September 2002, pp. 901-904 (2002)

19. Kneser, R., Ney, H.: Improved backing-off for $m$-gram language modeling. In: IEEE Int. Conf. on Acoustics, Speech and Signal Processing, vol. II, pp. 181-184 (May 1995)

20. Koehn, P.: Europarl: A parallel corpus for statistical machine translation. In: Proc. of the MT Summit X, pp. 79-86 (2005) 